Bei anderer Gelegenheit hatte ich das Wasser eines neu gegrabenen Brunnens zu untersuchen, welches sich als ganz ungemein stark eisenhaltig erwiesen hatte. Als ich die zum Eindunsten benutzte Platinschale zum weiteren Gebrauche dann mit verdünnter Salzsäure reinigte, bemerkte ìch einen für die geringe Rückstandsmenge sehr starken Chlorgeruch, der sich als von Mangan herrührend erwies. Dieser Umstand erklärte es auch, dafs dieses Wasser bei dem hohen Eisengehalte noch in Verbindung mit Mangan auf Wäsche massenhafte braune Flecken verursachte.

Ziemlich zu gleicher Zeit lagen mir viele Wasserproben zur Prüfung vor, die gleichfalls aus einem stark eisenhaltigen Gebiete herrührten; un rasch hierüber einen ungefähren Überblick zu erhalten, bediente ich mich des Mikroskops zum quantitativen Eisennachweis, indem ich je einen Tropfen auf dem Objektglas eindunstete und dann die Berlinerblaureaktion mittels Túpfelmethode anwandte und so für angeführten $Z_{w e c k}$ brauchbare Ergebnisse erhielt. Es bildeten sich hierbei so wunderschöne Bilder, dals dieselben als solche mit ihren feinen Äderchen und den zwar sehr kleinen, aber sehr scharf ausgesprochenen Krystallformen allein schon grofses Interesse beanspruchten.

Gewifs haben viele Kollegen ahnnliche besondere Ergebnisse bei Untersuchungen zu verzeichnen, und wäre es sehr zweckdienlich, wenn dieselben, angeregt durch meine Mitteilungen, solche auch veröffentlichen wollten.

Herrn Prof. Reichardt in Jena aber spreche ich bei dieser Gelegenheit meinen verbindlichsten Dank für manche praktischen Winke aus, die er mir im Laufe der Jahre in liebenswürdigster Weise bei verschiedenen Gelegenheiten gab, und dessen Grundlagen zur Beurteilung des Trinkwassers mich s.Z. zur Beschäftigung mit Wasseruntersuchungen veranlafsten.

\title{
Weitere Beiträge zur Kenntnis der Senegawurzel.
}

Von Ludwig Reuter, Apotheker, z. Z. in Heidelberg.

II.

In der letzten Arbeit habe ich gezeigt, dafs man bei der Wertbestimmung der Senegawurzel besonders den Gehalt derselben an fettem Öl und Harz, sowie das Vorhandensein ätherischen Öles, endlich auch den Feuchtigkeitsgehalt zu berifcksichtigen hat. 
Die Resultate der in dieser Hinsicht fortgesetzten Untersuchungen mit Material, welches mir von der weltbekannten Firma Thos. Christy in London freundlichst uberlassen wurde, teile ich in folgendem mit.

Zur Untersuchung standen zunächst drei lufttrockene Sorten zur Verfügung:

1. eine als "dry" (trocken) bezeichnete Wurzel, welche bei $110^{\circ} \mathrm{C}$. bis zar Gewichtskonstanz getrocknet immer noch 9,8 Proz. Feuchtigkeit und flüchtige Stoffe (ätherisches Öl) verlor. Sie bestand zum gröfsten Teil aus Wnrzeln von 20 bis $40 \mathrm{~mm}$ Dicke, die stärksten Wurzeln waren dicht unterhalb des Wurzelkopfes 0,5 bis $0,7 \mathrm{~cm}$ dick; die Kielung war an fast allen Exemplaren deutlich ausgeprägt. Wenn ich bei den Handelssorten der Senegawurzel bezilglich ihres früheren oder späteren Einsammelns (auf das Alter der Pflanze bezogen) drei Altersklassen unterscheiden darf, so möchte ich dio besprochene Wurzel in die mittlere Altersklasse einreihen. Die Farbe war ausgesprochen blarsgelb, der Geruch sehr schwach; es fehlte nicht an schönen sparrigen Exemplaren. Der Gehalt der lufttrockenen Wurzel an Salicylsturemethylester ergab sich zu 0,0033 Proz., der bei $1100 \mathrm{C}$. getrockneten zu 0,001 Proz. Das schwach grünlich-gelbe Ätherextrakt bestand zu 5,779 Proz. aus in Petroläther löslicbem fetten Öl und zu 1,521 Proz. aus in Petroläther unlöslichem Harz. Es ist beachtenswert, dafs in dieser Wurzel der Harzgehalt ein Viertel des Gehaltes an fettem Öl betrug.

2. Die zweite Sorte bestand aus einem Gemenge blafsgelber, sehr feiner, durchweg gebrochener, zum gröfseren Teile 2 bis $4 \mathrm{~cm}$, in einzeinen Fällen auch bis 6 und $7 \mathrm{~cm}$ langen, 1 bis $20 \mathrm{~mm}$ dicken Wurzeln mit dunkleren Wurzelköpfen. Letztere waren ziemlich grofs und umfangreich; die Wurzeln, dicht unterhalb des Kopfes bis $2,5 \mathrm{~cm}$ dick, waren nur 3 bis $4 \mathrm{~cm}$ lang, wobei in einzelnen Fällen die Länge des eigentlichen Kopfes allein schon bis $2 \mathrm{~cm}$ betrug. Im allgemeinen repräsentierte sich diese Sorte als fast durchweg gebrochene Ware; vollständigere Wurzeln, aus Wurzelkopf und längeren Wurzeln nebst Nebenwurzeln bestehend, waren nicht vorhanden. Der Gehalt dieser Wurzel an Feuchtigkeit und flüchtigen Stoffen warde za 10,1 Proz., der Gehalt an Salicylsäuremethylester in der luff- 
trockenen Wurzel zu 0,125 Proz., in der bei $110^{\circ}$ getrockneten zu 0,025 gefunden. Dis lufttrockene Wurzel lieferte 7,1 Proz. Ätherextrakt, davon 6,454 Proz. fettes Öl und 0,646 Proz. Harz. Der Harzgehalt betrug somit in diesem. Falle nur ein Zehntel des Gehaltes der Wurzel an fettem Öle.

3. Die dritte Sorte hatte im allgemeinen ein besseres Aussehen, als die beiden bereits besprochenen. Die Menge der vorhandenen Wurzelköpfe sowie die Dicke derselben entsprach der Menge der vorhandenen Wurzeln und Nebenwurzeln. Der Farbe nach bestand diese Sorte aus einem Gemenge heller blafsgelber und etwas dunklerer Ware. Der Kiel war sehr scharf ausgeprägt. Dle Dicke der stärksten Wurzeln dicht unterbalb des Wurzelkopfes betrug da und dort 0,7 bis $1 \mathrm{cre}$, vorwiegend aber nur 20 bis $40 \mathrm{~mm}$. Ein Exemplar war insofern interessant, als die vom Wurzelkopf ausgehende Wurzel in ahnlicher Weise gekrïmmt, wie man dies bei Rhizoma Bistortae fast regelmäfsig zu beobachten Gelegenbeit hat.

Diese Sorte besals einen ziemlich starken Geruch; bei $110^{\circ} \mathrm{C}$. getrocknet, verlor sie 10,5 Proz.; lufttrocken ergab sie einen Gehalt von 0,45 Proz., bei $110^{\circ}$ C. getrocknet einen solchen von 0,08 Proz. Salicylsäuremethylester. Der Verdunstungsrückstand des ätherischen Auszuges besals eine schön goldgelbe Farbe und bestand aus 5,748 Proz. fettem Öl und 0,554 Proz. Harz. Es ist nicht uninteressant, neben den beschriebenen drei Sorten englischer Provenienz noch zwei deutsche Handelssorten - eine als "electissima“, die zweite als "depurata" bezeichnet zu betrachten.

Die electissima - in welcher ich, nebenbei erwähnt, auf $100 \mathrm{~g}$ ein kleines Stïckchen (ca. 0,8 g) einer Wurzel von Cypripedium parviflorum finde - besteht aus 10,15, 20, ja sogar bis $25 \mathrm{~cm}$ langen, dicht unter dem Wurzelkopf höchstens 20 bis $25 \mathrm{~mm}$, vorwiegend nur bis $20 \mathrm{~mm}$ dicken.Hauptwurzeln mit sehr zarten und langen Nebenwurzeln, welche den Hauptwurzeln noch anhaften. Der Kiel ist - der Grölse und Dicke der Wurzel entsprechend - recht schön ausgeprägt; einzelne lose Wurzelköpfe sind nicht vorhanden, sondern jedem derselben entspringen ziemlich lange, oft sparrige, Wurzeln nebst Nebenwurzeln. Diese Sorte repräsentiert jedenfalls insofern eine vollkommene Wurzel, als eine verhältnismärsig grofse Menge von Rindenteilen vorhanden ist, welche nach Schneider einen gröfseren Senegingehalt haben, als die inneren 
Teile der Wurzel. Die Farbe ist eine blasse bei den feineren Wurzelteilen, eine etwas dunklere bei den gröfseren und alteren Wurzeln. Jedenfalls ist diese Sorte sehr jung gesammelt, was schon aus dem Vorhandensein nur zarter, kleiner Wurzelköpfe hervorgeht. Der Kiel ist an allen Exemplaren deutlich sichtbar.

Ganz anders stellt sich die zweite Senøgasorte deutscher Provenienz vor, die als "depurata" bezeichnet ist. Sie macht einen massigen Eindruck, ist viel dunkler und besteht zum gröfseren Teile aus sehr dicken und grofsen Wurzelköpfen, von welchen nur bis $3 \mathrm{~cm}$ lange Wurzeln auslaufen. Wie merkwürdig manchmal die Handelsbezeichnungen von Drogen gewählt werden, ist aus der Bezeichnnng der beiden deutschen Handelssorten ersichtlich. Beide sind sich volltg ebenbürtig hinsichtlich ihrer Reinheit, trotzdem heilst die eine ,electissima", die andere „depurata". Aus dem pharmakognostischen Studium geht aber hervor, dafs die Wurzeln beider Sorten in ganz verschiedenen Altersstadien gesammelt sind. Das Alter der Wurzel zur. Zeit der Einsammlung und der dem Alter entsprechende Bau der Wurzel dtirfte besonders bei der Senegawurzel allein mafsgebend sein zur Feststellung einer Handelsbezeichnung, aus welcher der Apotheker sofort orsehen kann, auch wenn er nur die Preisliste einer Firma zur Hand hat, welche Sorte er unter dieser oder jener Titulatur bekommt. Jedenfalls dürfte sich die Einfuhrung von Bezeichnangen wie Radix Senegae depurata capitibus majoribus, Radix Senegae depurata capitibus minoribus empfehlen; letztere entspräche dann der "electissima", erstere der "depurata".

Die Untersuohung der beiden Sorten bezïglich des Verlustes beim Trocknen bei $110^{\circ} \mathrm{C}$, dann des Gehaltes an fettem Öle, Harz und Salicylsäuremethylester ergab folgende Werte:

a.

I. electissima. II. depurata.

Verlust beim Trocknen .... 6 Proz. 7,6 Proz.

Äther-Extrakt $(\ddot{O} l+\mathrm{Harz}) \ldots 7,2, n \quad 7$

Hiervon fettes $\mathrm{Ol} \ldots \ldots \ldots, 6,55 n, 6,35 n$

Harz ........ 0,62. , 0,6 ,

b.

Mit Petroläther extrahiert: I. electissima. II. depurata.

1. direkt durch einf. Maceration, fettes $\mathrm{Öl} \ldots \ldots \ldots$. 6,6 Proz. 6,3 Proz.

2. in Soxleth's Apparat, fettes ÖI 6,7 n $\quad 6,82$ n 
456 Ludwig Revter, Weitere Beiträge zur Kenntnis d. Senegawurzel.

c.

Gehalt an Salicylsåuremethylester

I.

Ш.

vor dem Trocknen der Wurzel

0,13 Proz.

0,16 Proz.

nach dem Trocknen der Warzel

$0,01 "$

0,025 ,

Aus dem Vaterlande eines Pereira und Hanbury erhielt ich durch die Güte des Herrn Richard Bremridge, Secretary of the Pharmaceutical Society of Great Britain, und des Herrn E. M. Holmes, Curator of the Society Museum, verschiedene Sorten von Senega, und zwar:

1. Root of Polygala Senega var. latifolia Northern Senega,

2. Genuine Senega of London commerce,

3. Prime Southern Senega from New York,

4. Prime Western Senega from New York,

5. Larger root found in ordinary Senega of commerce. Intermediate in size between it and Northern Senega.

6. False Southern Senega of Polygala Boykinii Nuttal.

Durch Vergleich habe ich gefunden, dafs die in meiner ersten Arbeit „über die Senega" abgehandelte nördliche (nordwestliche) Senega mit der vom Pharmaceutical Museum in London erhaltenen Northern Senega ubereinstimmt. Der Kiel ist hier wie dort nur ausnahmsweise vorhanden. - Die früher besprochene südliche (südwestliche) Senega entspricht der von Herrn E. M. Holmes erhaltenen "Genuine Senega of London commerce ${ }^{\mu}$.

Die "False Senega of Polygala Boykinii" ist es nun, auf welche ein ganz besonderes Augenmerk zu richten ist. Prof. John M. Maisch, dessen Name mit der Geschichte der Pharmacie in den Vereinigten Staaten eng verknüpft ist und überall da einen guten Klang hat, wo es sich um die Interessen unserer Wissenschaft bandelt, teilt mir in einem freundlichen Briefe (vom 16. März a. c.) über die Verwechselungen der Senegawurzel folgendes mit:

„Eine Verfälschung der Senega findet hier böchst selten statt, doch finden sich zuweilen in gröfseren Mongen Wurzeln anderer Pflanzen, welche denselben.Standort haben. Eine leicht tăuschende Verfälschung, welche ich vor einer Reihe von Jahren beobachtete, bestand in den Nebenwurzeln einer oder mehrerer hiesigen Species von Gentiana; welche in Grölse, Farbe und sonstigem Aussehen viel Ahnlichkeit mit Senega haben, auch stärkefrei sind, sich aber durch die Struktar und 
Ludwig Reuter, Weitere Beiträge zur Kenntnis d. Senegawurzel. 457

den Geschmack leicht unterscheiden lassen, dagegen findet sich zeitweise - so wieder 1888 - eine falsche Senega in grölseren Mengen im Handel, welche wahrscheinlich von Polygala Boykinii in unseren Südwest-Staaten stammt, ganz den Wuchs der Senega hat, jedoch mehr gerade und weniger sparrig wăchst, einen runden Holzkörper besitzt und in getrocknetem Zustande nicht den Kiel zeigt, sie hat der Senega ähnliche Bestandteile und Eigenschaften, ist aber von schwächerer Wirkung. ${ }^{*}$

In dem von Prof. J. M. Maisch im "National Dispensatory" geschriebenen Artikel "Senega“ lesen wir aufser dem schon erwähnten über die falsche Senega noch folgendes:

"White or false senega was quite common in the market for several years after 1875. It was said to have been collected in Southwestern Missouri. This root yielded to Goebel (1881), by Quevennes process, 3 Proz. of Senegin, and all preparations wade with it were much lighter in color, than the corresponding preparations of true senega. Another kind of so called white senega, which we have seen was amylaceous internally, and bad none of the prominent characteristics of the pharmacopocial drug."

Wie aus dem Mitgeteilten zu ersehen ist, besitzt die von Polygala Boykinii stammende Wurzel so charakteristische makroskopische Merkmale, dafs eine Vermischung mit der offizinellen Ware sich leicht und sicher feststellen läfst, freilich nur bei ganzer Ware. Ob die falsohe Senega von der echten differierende chemisch nachweisbare Bestandteile hat und in geschnittener Wurzel ein zweifelloser Nachweis derselben ausgefuhrt werden kann, dariber werde ich erst Mitteilungen machen können, wenn ich in den Besitz des nötigen Versuchsmaterials gelangt bin und diesbezügliche Untersuchungen bethätigt sind.

Durch die Gite des Herrn Dr. Friedrich Hoffmann in New York, jenes bedeutenden Kollegen, dessen Leben von kompetenter Feder vor noch nicht langer Zeit in einem unserer deutschen Fachblätter skizziert wurde, bin ich soeben in den Besitz verschiedener, zweifellos echter Senega-Muster gelangt, über welche ich mir zum Schlusse meiner heutigen Mitteilungen noch zu referieren erlaube.

Herr Dr. Hoffmann batte die Liebenswürdigkeit, mir zu senden:

1. eine vollständige Pflanze von Polygala Senega aus Indiana stammend, 
2. südliche und westliche Senegawurzel von Westrirginien,

3. südliche und westliche Senega von Kentucky,

4. südliche und westliche Senega,

5. nördliche Senega von Wisconsin.

Die südliche und westliche Senegawurzel aus Westvirginien ist von blafsgelber Earbe, die ans Kentucky stammende etwas dunkler; erstere ist in viel jüngerem Zustande gesammelt als letztere, bei beiden fehlt der Kiel fast durchweg.

Die ohne nähere Bezeichnung unter 4 angeführte südliche und westliche Senega ist dunkler als die aus Kentucki stammende, jedoch gekielt.

Die nördliche Senega aus Wisconsin besteht aus aufserordentlich voluminösen, knorrigen Wurzelköpfen von 3 bis $4 \mathrm{~cm}$ Durchmesser, weichen nur bis $5 \mathrm{~cm}$ lange, an der dicksten Stelle $2,5 \mathrm{~cm}$ dicke, am dunnsten Ende $0,8 \mathrm{~cm}$ dicke Wurzeln entspringen.

Die unter 1 aufgefthrte vollständige Pflanze:von Polygala Senega hat Wurzeln, die sehr schön gekielt sind und in jeder Hinsicht der "Genuine Senega of London commerce" gleichen.

Über die chemische Untersuchung der von Herm Dr. Hoffmann erhaltenen Muster werde ich das nächste Mal berichten, heute möchte ich zum Schlusse nur noch darauf hinweisen, dafs minimale Beimengungen von Cypripediumwurzeln zur Senega kaum von bemerkenswertem Einflusse auf die Wirkung der letzteren sein dirften. Cypripedium ist eine jener Drogen, die, schon von den nomadisierenden Eingeborenen Nordamerikas als Heilmittel angewandt, in den Gebrauch des ansässigen amerikanischen Landvolkes uberging und von demselben bei nervösen Affektionen, besonders in Fällen von Chorea und Hysteria, heute noch als Arzeneimittel geschätzt ist. Die einzige im Jahre 1886 von H. C. Blair ausgeführte Untersuchung erwies die Anwesenheit von Spuren ätherischen Öles, einer flüchtigen Säure, zweier Harze, Gerbstoff und Stärke.

Die Wirkung soll der der Valeriana ähnlich sein.

Die von mir der Destillation unterworfene Cypripediumwurzel, welche ich ebenfalls der Liebenswürdigkeit des Herrn Dr. Fr. Hoffmann verdanke, gab ein Destillat, das indifferent gegen Fisenchlorid, somit frei von Salicylsäuremethylester war, während die genannte Verbindnng, wie ich wiederholt gezeigt habe, ein wichtiger Anhaltspunkt ist zur Identifizierung frischer Senega. Auch das Destiliat der Ginsengwurzel, welche in meiner ersten Mitteilung über die Senega als zu- 
fallige Beimischung derselben Erwähnong gefunden, erwies sich frei vom erwähnten Salicylsäureester. Die Ginsengwurzel enthält jedoch Panaquilon, einen dem Glycyrrhizin ähnlichen, von Garrigues zuerst beschriebenen Stoff, welcher dadurch charakterisiert ist, dafs er sich nit konzentrierter $\mathrm{H}_{2} \mathrm{SO}_{4}$ herrlich purpurrot färbt. Mit Hilfe dieser Reaktion läfst sich die Ginsengwurzel auch in Dekokten, die freilich zu konzentrieren sind, nachweisen.

Für heute schliefse ich mit dem Vorschlage, der von kompetenter Hand gegebenen Fassung des Artikels "Senega" der deutschen Reichspharmakopöe einen kleinen Zusatz für die werdende neue Pharmakopöe beizufingen, welcher das Alter resp. den Gehalt der Wurzel an Salicylsäuremethylester berücksichtigt und vielleicht in folgende Worte $\mathrm{zu}$ kleiden wäre: ${ }^{5} \mathrm{~g}$ lufttrockener Senegawurzel mit $50 \mathrm{ccm}$ Wasser von ca. $60^{\circ} \mathrm{C}$. ibergossen, muls nach 15 Minuten ein Filtrat geben, welches mit 3 Tropfen Salzsäure angesäuert und mit $50 \mathrm{ccm} \ddot{A} t h e r$ a usgeschittelt, an letzteren soviel Salicylsäure abgibt, dafs nach Aufnahme-der freiwillig verdunsteten ätherischen Ausschattelung mit $20 \mathrm{ccm}$ Wasser von $600 \mathrm{C}$. auf Zusatz eines Tropfens Eisenchloridlösung eine deutlich violette Farbenreaktion eintritt."

Uber die eventuelle Verwertung einer Harz- und Fettbestimmung mlissen weitere Daten gesammelt werden.

\section{Steinholz.}

Von Dr. E. Pfeiffer in Jena.

Das einer Dresdener Firma patentierte sogen. Steinholz, welches zur leichteren Herstellung tropischer Wohnungen unter Anwendung lediglich eiserner Verbindungssticke empfohlen ist, besteht aus dem bekannten Magnesiacement, also (etwa) $\mathrm{MgCl}_{2}, 5 \mathrm{MgO}+14 \mathrm{H}_{2} \mathrm{O}$ im Gemenge mit Sägemehl. - Auf der Wiener Weltansstellung würde als Kajalith eine allgemein bewunderte, schön polierte Tischplatte prämïrt, welche von Dresden gesandt, aus Gyps durch Wasserglas gehärtet, hergestellt sein sollte; als sie sich aber, dem Einflufs der Witterung ausgesetzt, nicht standhaft erwies, fand sie sich, bei der nun erst folgenden Untersuchung, als aus Magnesiacement bestehend. Wie das Dazukommen 